\title{
Isolation of Escherichia coli and Salmonella spp. from free-ranging wild animals
}

\author{
Renata de Oliveira Iovine ${ }^{1}$, Catia Dejuste ${ }^{2,3}$, Flávia Miranda ${ }^{2}$, Claudia Filoni ${ }^{1,3,4}$, \\ Marina Galvão Bueno ${ }^{3,5}$, Vania Maria de Carvalho ${ }^{1}$ \\ ${ }^{1}$ Laboratório de Biologia Molecular e Celular, Universidade Paulista, São Paulo, SP, Brazil. \\ ${ }^{2}$ Projeto Tamanduá, São José dos Pinhais, PR, Brazil. \\ ${ }^{3}$ Instituto Brasileiro para Medicina da Conservação, Recife, PE, Brazil. \\ ${ }^{4}$ Laboratório de Diagnóstico Molecular, Universidade Estadual Paulista "Julio de Mesquista Filho", \\ Botucatu, SP, Brazil. \\ ${ }^{5}$ Laboratório de Patologia Comparada de Animais Selvagens, Universidade de São Paulo, São Paulo, \\ SP, Brazil.
}

Submitted: October 1, 2014; Approved: April 16, 2015.

\begin{abstract}
Increasing interactions between humans, domestic animals and wildlife may result in inter-species transmission of infectious agents. To evaluate the presence of pathogenic E. coli and Salmonella spp. and to test the antimicrobial susceptibility of isolates, rectal swabs from 36 different free-ranging wild mammals were taken from two distinct natural sites in Brazil: Cantareira State Park (CSP, state of São Paulo) and Santa Isabel do Rio Negro Region (SIRNR, state of Amazonas). The swabs were randomly collected and processed for bacterial isolation, identification, characterization and antimicrobial resistance. Eighteen E. coli strains from CSP and 20 from SIRNR were recovered from 14 and 22 individuals, respectively. Strains from animals captured in CSP, the site with the greatest anthropization, exhibited a higher range and percentage of virulence genes, including an $e a e+/ b f p A+$ strain. Antimicrobial resistance was verified in strains originating from both sites; however, in strains from SIRNR, aminopenicillins were almost the exclusive antimicrobial class to which strains exhibited resistance, whereas in CSP there were strains resistant to cephalosporins, sulfonamide, aminoglycoside, tetracycline and fluoroquinolone, in addition to strains exhibiting multidrug resistance. Two strains of Salmonella enterica that are known to be associated with reptiles, serotypes Belem and 60:r:e,n,z15, were recovered only from Amazonian animals and showed susceptibility to all classes of antimicrobials that were tested. Although the potential impact of these pathogens on wildlife remains unknown, bacteria isolated from free-ranging wild animals may provide relevant information about environmental health and should therefore be more deeply studied.
\end{abstract}

Key words: E. coli, Salmonella, wild mammals, One Health, Brazil.

\section{Introduction}

Wildlife plays an important role within the context of the One World, One Health concept. Wild animals can serve as sentinels for infectious diseases and illnesses resulting from environmental contamination with toxic substances; however, wildlife can also transmit pathogens to other animal species (directly and indirectly through environmental dispersal), including humans (Rabinowitz et al., 2009). Escherichia coli is one of the primary intestinal commensal organisms found in endothermic animals and therefore it is widely disseminated in the environment. Investigating potential pathogenic $E$. coli strains and/or antibiotic resistance may provide information regarding human activity in select ecological niches. Studies have indicated that living in proximity to humans may alter the E. coli populations of wildlife intestinal microbiota (Rwego et al., 2008; Gordon and Cowling, 2003), which can in turn serve as a reservoir of $E$. coli strains that are pathogenic to humans (Bélanger et al., 2011). 
Bacteria belonging to the Salmonella genus are recognized as important primary zoonotic pathogens. Unlike E. coli, Salmonella spp. are capable of persisting in aquatic environments for long periods of time, even in locations with high levels of eutrophication. The ubiquitous nature of Salmonella permits a cyclic exchange between host and environment (Winfield and Groisman, 2003). Thus, wildlife can serve as reservoirs of diverse serotypes, especially with respect to reptilian species (Winfield and Groisman, 2003; Hoelzer et al., 2011).

Antimicrobial resistance in bacteria isolated from individuals with no history of previous exposure to antimicrobial agents is indicative of the environmental dissemination of resistant strains and residual antimicrobial products through effluents from urban areas and animal production units (Costa et al., 2013). The present study was conducted to evaluate the presence of pathogenic E. coli and Salmonella spp. and to test antimicrobial susceptibility in isolates taken from fecal samples of free-ranging wild mammals in two distinct natural sites in Brazil.

\section{Materials and Methods}

\section{Animals and biomes}

A total of 14 different wild mammals (Table 1) from Cantareira State Park (CSP) (10 coati, 3 opossum, and 1 grey short-tailed opossum) and 22 (Table 2) from the Santa Isabel do Rio Negro Region (SIRNR) (18 bats, 3 rodents and 1 marsupial), all of them apparently healthy, were randomly captured using traps and were chemically restrained in strict accordance with protocols approved by the Research Ethics Committee (Permit Number: 140/12 $\mathrm{CEP} / \mathrm{ICS} / \mathrm{UNIP}$ ). All activities were performed in full compliance with federal permits issued by the Brazilian Ministry of Environment (Permit Numbers: CSP260108-000.327/0 2008; SIRNR- SISBIO 27923-4). CSP $\left(23^{\circ} 26^{\prime} 58.84^{\prime \prime} \mathrm{S} ; 46^{\circ} 38^{\prime} 7.94^{\prime \prime} \mathrm{O}\right)$ is located in one of the world's largest native areas of Atlantic forest within a metropolitan area (the city of São Paulo, which is the largest metropolis in South America), allowing for the possibility of close interactions between local fauna and visitors and domestic animals inhabiting the surrounding areas. In contrast, SIRNR $\left(0^{\circ} 24^{\prime} 51.61^{\prime \prime} \mathrm{S} ; 65^{\circ} 1^{\prime} 10.23^{\prime \prime} \mathrm{O}\right)$ is located in Amazonia and is accessible only via fluvial transportation. Human population density is low in this region.

\section{Bacterial isolation and identification}

Two rectal swabs were taken per animal. The swabs were delicately introduced into the rectum through rotational movements after cleaning the perianal area with gauze and sterile saline. The swabs were then immediately placed in Stuart transport medium (COPAN Venturi Transystem $\left.{ }^{\circledR}\right)$ and kept under refrigeration. The first swab that was collected was seeded onto MacConkey Agar (Difco) to isolate E. coli, and the resulting colonies were identified using Enterokit B (Probac do Brasil). To isolate Salmonella spp., the second fecal swab sample was processed according to a previously published protocol (Michael et al., 2003). Colonies suggestive of Salmonella spp. were confirmed using an API 20E identification system (BioMerieux), and they were serotyped in the Reference Center for Salmonella Serotyping (Instituto Adolfo Lutz, São Paulo, SP, Brazil).

\section{E. coli virulence genotype and phylogenetic analysis}

Strains identified as E. coli that were phenotypically different from each other were frozen in Brain Heart Infusion Medium (Difco) with $80 \%$ glycerol [Invitrogen; $1: 1(\mathrm{v} / \mathrm{v})]$ at $-80^{\circ} \mathrm{C}$ for further analysis. A PCR assay was used to test the isolates for the presence of virulence genes (VGs) known to be associated with diarrheagenic E. coli (DEC) strains (eae, bfpA, stxl and stx2) (Pollard et al., 1990; Olsvik et al., 1991; Gannon et al., 1993; Gunzburg et al., 1995) and with extraintestinal pathogenic E. coli (ExPEC) (papC, papEF, sfa, fyuA, iucD, hly, cnfl, cvaC, traT and $\mathrm{malX}$ ) (Le Bouguenec et al., 1992; Johnson and Stell, 2000). Phylogenetic groups were determined using an established PCR-based method for assessing chuA and yjaA genes and the TSPE4.C2 DNA fragment (Clermont et al., 2000).

An EDL 933 strain was used as a positive E. coli control for eae, stxl, and stx2 genes, and an E2348/69 strain was used as the control for the $b f p A$ gene. For ExPEC genes, C7, JJ079 and BUTI 1-7-6 strains were used as positive controls in all PCR reactions, and the JJ055 (derived from K12) strain was used as a negative $E$. coli control (all were provided courtesy of Prof. Dr. James R. Johnson, University of Minnesota). The controls used for phylogenetic determination were JJ055 (Group A), EC 15 (Group B1), JJ079 (Group B2), and EDL 933 (Group D).

\section{Antimicrobial susceptibility test}

Antimicrobial susceptibility was assessed using a disk diffusion method according to CLSI protocols (CLSI, 2013a; CLSI, 2013b). The following antimicrobials were tested: the amoxicillin $(10 \mu \mathrm{g})$ and ampicillin $(10 \mu \mathrm{g})$ aminopenicillins; the cephalexin $(30 \mu \mathrm{g})$, cefoxitin $(30 \mu \mathrm{g})$ and ceftiofur $(30 \mu \mathrm{g})$ cephalosporins; the ciprofloxacin $(5 \mu \mathrm{g})$ and enrofloxacin $(5 \mu \mathrm{g})$ fluoroquinolones; the amphenicol chloramphenicol $(30 \mu \mathrm{g})$; the combined sulfonamide sulfametaxazol + trimethoprim $(25 \mu \mathrm{g})$; the streptomycin $(10 \mu \mathrm{g})$ and gentamicin $(10 \mu \mathrm{g})$ aminoglycosides; and tetracycline $(30 \mu \mathrm{g})$. Strains resistant to three or more classes of antimicrobials were considered to be multidrug resistant (MDR) (Schwarz et al., 2010). 


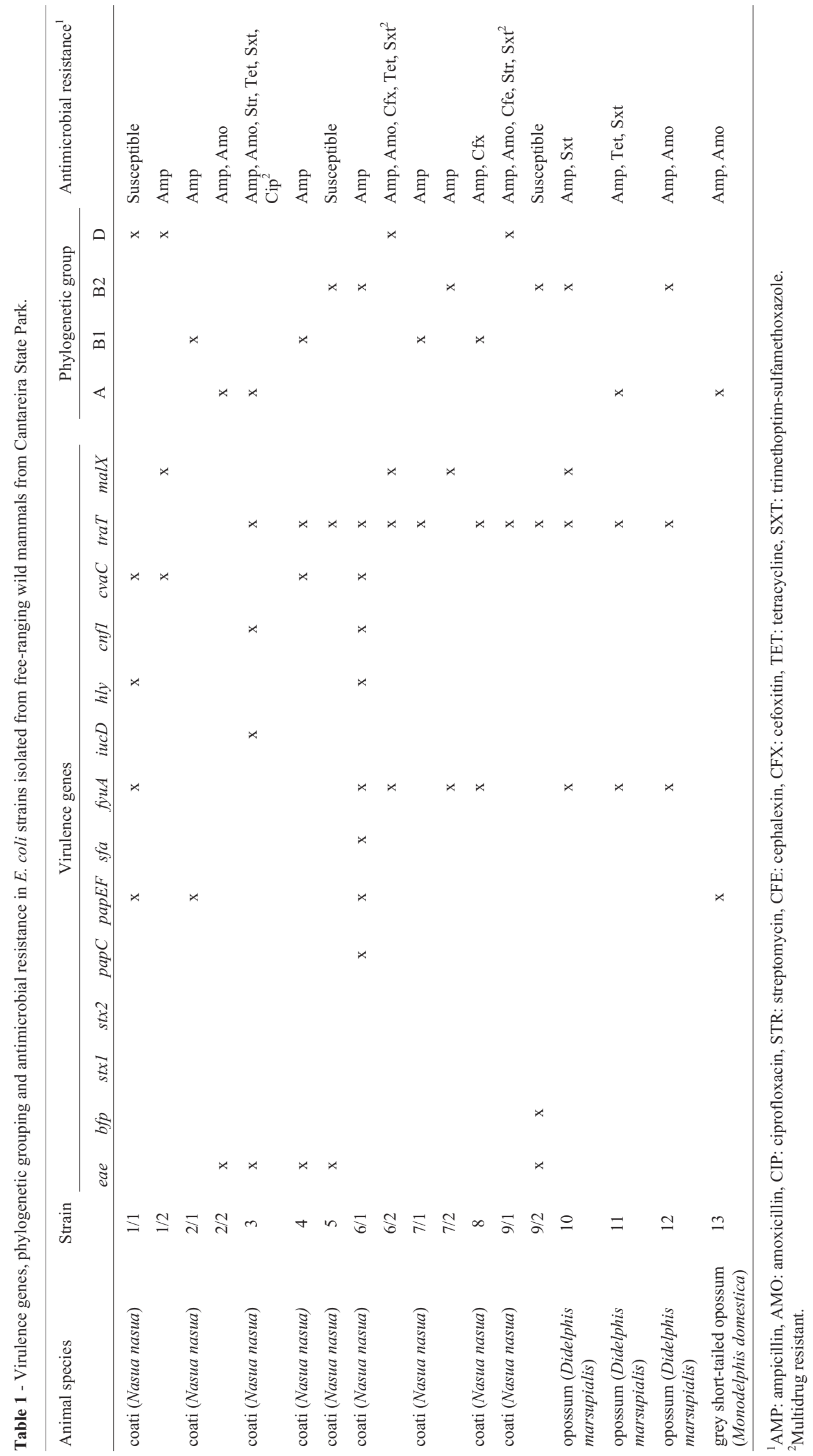




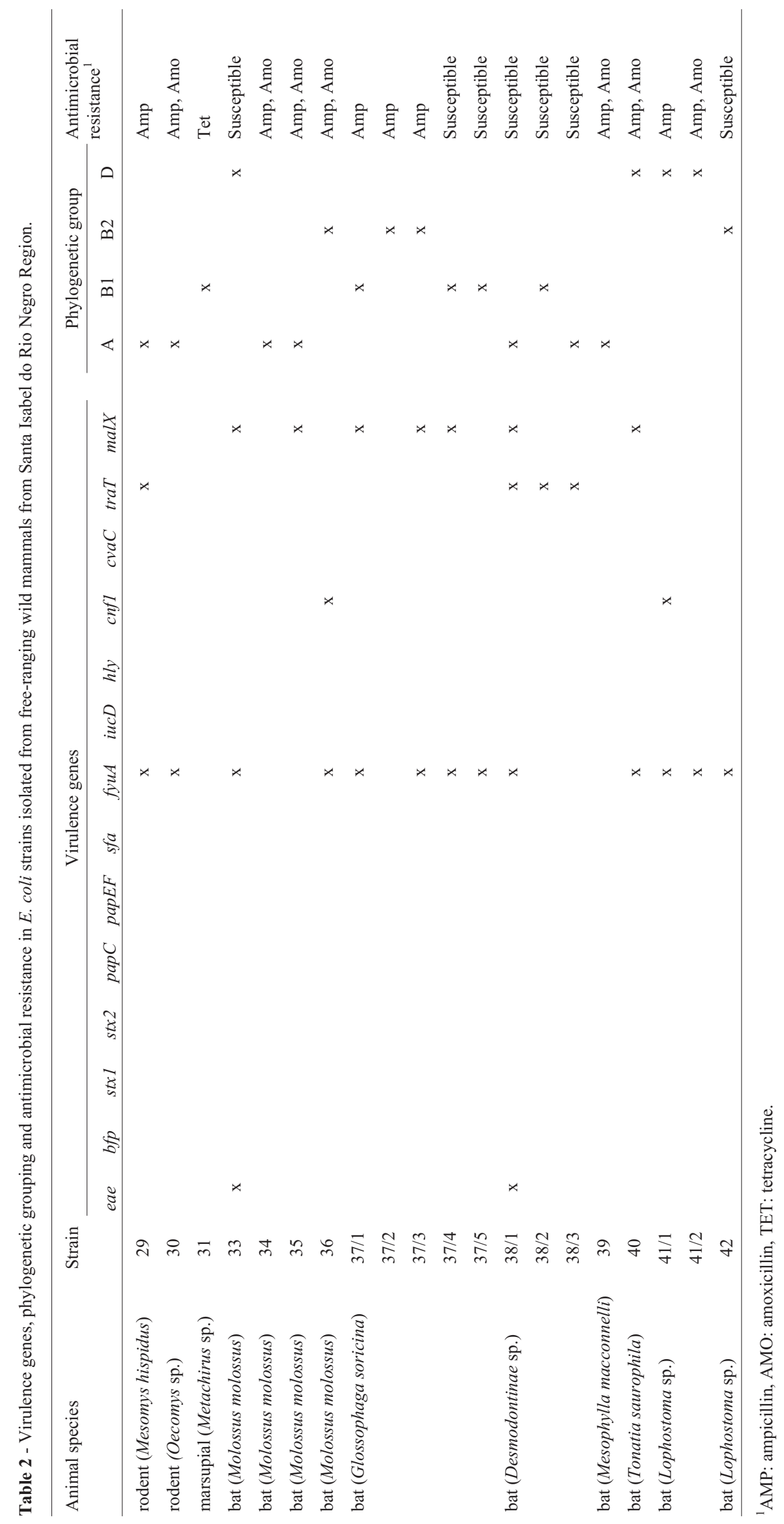




\section{Results and Discussion}

\section{Isolation and prevalence of $\mathrm{VG}$ and phylogenetic groups in E. coli}

E. coli strains were isolated in 13 out of $14(92 \%)$ and 13 out of $22(59 \%)$ of the animals from CSP and SIRNR, respectively. The occurrence of $E$. coli in the mammalian gut can vary enormously, but typically $E$. coli makes up more than $90 \%$ of gut microbiota in humans and only $56 \%$ of gut microbiota in wild mammals (Gordon and Cowling, 2003; Tenaillon et al., 2010). The recovery rate of E. coli was higher in animals from CSP, which may reflect a more intense exposure to human contamination of the environment. According to Gordon and Cowling (2003), the probability that a given host is carrying $E$. coli depends on the frequency of exposure, the likelihood that an exposure would result in the establishment of a population and, finally, the average period of time that an E. coli population could remain in a host. These issues are relevant when evaluating the importance of wildlife interactions with humans. Therefore, a total of 18 and 20 E. coli strains were successfully isolated from mammals in CSP and SIRNR, respectively. Characterizations of these strains are summarized in Tables 1 and 2.

This study verified that in the majority of $E$. coli isolates from CSP, both the number and range of VGs were higher than in those isolated from SIRNR; the same was true for antimicrobial resistance patterns. Whereas in SIRNR only two out of 20 isolated strains $(10 \%)$ contained three or more VGs, in CSP seven out of 18 (39\%) showed this profile. Considering that $E$. coli VGs are rare in the microbiota of wild animals but are not rare in humans (Tenaillon et al., 2010) and that the domestication status (which can be defined as living in proximity with humans beings) of an animal host is the principal environmental force that shapes the genetic structures of commensal $E$. coli populations (Escobar-Páramo et al., 2006), strains recovered from wild animals in CSP would reflect a broader anthropogenic action.

This hypothesis may be reinforced by the fact that the eae gene was only present in $10 \%(2 / 20)$ of strains in animals taken from SIRNR and in 28\% (5/18) of those taken from CSP. However, none of the isolates were positive for either the $s t x 1$ or $s t x 2$ genes. The eae $+/ s t x$ - markers are associated with the DEC, attaching and effacing of E. coli, and although strains with these markers are recognized as pathogens for humans and domestic animals, few studies have described their presence in wildlife (Bardiau et al., 2010; Obwegeser et al., 2012). Although a complete characterization of the eae+ strains that were found in this survey has not yet been performed, a search for $b f p A$ genes revealed one strain from CSP with the profile eae+/bfp $A+$. CSP is a natural area with a high anthropogenic impact, and isolates with this profile are linked to typical EPEC, which are strains that have been epidemiologically associated with human beings (Trabulsi et al., 2002). It is important to acknowledge that the strain was isolated from a coati, specie that actively interacts with park visitors and their waste.

Furthermore, approximately $33 \%(6 / 18)$ of the strains isolated from CSP samples had some combination of the following genes: $p a p E F, c v a C$, traT and $m a l X$. All of these genes have been correlated with the pathogenic potential of ExPEC (Johnson et al., 2001; Maynard et al., 2004); however, only a single strain (5.5\%) from SIRN exhibited similar findings ( $m a l X$ and traT). Previous studies have indicated that the presence of different combinations of VGs may provide a competitive advantage for the establishment of bacteria in intestinal microbiota as well as in the development of extra-intestinal infections (Diard et al., 2010). P fimbria genes, such as papC and $p a p E F$, are important indicators of pathogenicity and have been associated with isolates that cause urosepsis in pets and humans (Maynard et al., 2004). The papEF gene was present in $22 \%(4 / 18)$ of the strains isolated from CSP animals, but it was absent in SIRNR isolates. The $c v a C$ gene is linked to $\mathrm{ColV}$, a plasmid that occurs significantly more frequently in ExPEC recovered from neonatal meningitis compared to commensal E. coli (Johnson et al., 2010; Bélanger et al., 2011), and it was likewise found exclusively in the strains isolated from CSP mammals. The gene traT encodes a cell surface lipoprotein that increases bacterial resistance to the lytic actions of complement (Johnson and Stell, 2000). This gene was found in $67 \%(12 / 18)$ of CSP strains and only $20 \%(4 / 20)$ of SIRNR isolates.

The malX gene, a pathogenicity island (PAI) marker of the archetypal uroseptic strain CTF073 (06:K2:H1), is associated with urinary tract infections in both humans and pets, in addition to human pyelonephritis, bacteremia and neonatal meningitis (Johnson et al., 2001; Johnson et al., 2003). Surprisingly, this gene was found in $35 \%$ of SIRNR strains (7/20) and in $22 \%(4 / 18)$ of CSP strains. The discovery of this gene even in isolates from a less impacted environment may be due to the fitness demonstrated by strains possessing PAIs. As these genetic elements increase the competitive abilities of their carriers in the intestinal environment compared to isolates in which they are absent, they contribute to the intestinal persistence of $E$. coli (Östblom et al., 2011; Diard et al., 2010). Thus, if through it's gregarious behavior a wild animal acquires an E. coli strain that is harboring this gene, the presence of this marker could subsequently be amplified within the animal population. Out of all of the animals that were examined in this study, half of bats were found to harbor E. coli malX+. This is notable, as bats are characterized by living in large colonies and have the ability to travel through diverse ecological niches (Mühldorfer, 2013).

A phylogenetic classification indicated that $56 \%$ $(10 / 18)$ of the strains isolated from CSP belonged to phylogenetic groups B2 and D, whereas in SIRNR this percentage was slightly lower $(40 \%, 8 / 20)$. The majority of 
commensal strains are derived from phylogenetic groups A and $\mathrm{B} 1$, whereas pathogenic strains generally belong to groups B2 and D. Group B2 is largely composed of ExPEC, which is a heterogeneous group of strains that are able to asymptomatically colonize the intestines of several different hosts (Smith et al., 2007). Most importantly, a B2 strain that contained eight of the ExPEC genes that were included in our study was only isolated from animals in CSP. These genes encoded for adhesins ( $p a p C / p a p E F$ and $s f a$ ), an iron capture system $(f y u A)$, toxins (hly and cnfl), protectin (traT) and a ColV associated-virulence plasmid ( $\mathrm{cvaC})$ (Smith et al., 2007; Bélanger et al., 2011). Although the pathogenicity of these strains to wild animals is unknown, we can hypothesize that these animals were exposed to sources of potentially pathogenic bacteria because extraintestinal VGs are rare in isolates from wildlife, as has been previously described (Tenaillon et al., 2010).

\section{Antimicrobial susceptibility}

The presence of resistant bacteria in animals that had not been directly exposed to antimicrobials may reflect the degree of local anthropization (Costa et al., 2013). A total of $83 \%(15 / 18)$ of the strains isolated from CSP and $65 \%$ of those isolated (13/20) from SIRNR demonstrated resistance to one or more antimicrobial agents. However, isolates from SIRNR were almost exclusively resistant to aminopenicillins, whereas strains from CSP were resistant to cephalosporins, sulfonamide, aminoglycoside, tetracycline and fluoroquinolone, in addition to presenting multidrug resistance (Table 1 and 2). Among the drugs tested, chloramphenicol was the only antimicrobial agent to which all of the strains that were recovered from CSP were susceptible. It is interesting to note elevated aminopenicillin resistance indices were found in SIRNR strains (12 out of 20 strains), ten of which were isolated from bats. These drugs are widely used in the treatment of human disease, as well as in companion and production animals (Maynard et al., 2004), and their active compounds remain almost completely intact when they are eliminated into the environment. This may lead to environmental contamination and a dissemination of antimicrobial resistance (Costa et al., 2013). Because SIRNR has fewer human and domestic animal residents, the results suggest that the observed resistance phenomenon was not limited to its original niches and that wildlife may have an important role in its spread. Bats are capable of traveling through wide geographic distances and diverse ecological niches; furthermore, their colonial population pattern facilitates the sharing of strains (Mühldorfer, 2013).

\section{Salmonella isolation}

Two strains of Salmonella enterica, serotype Belem and subspecies diarizonae serotype 60:r:e,n,z15, were respectively isolated from a bat and a rodent; both serotypes have been previously associated with reptiles (Schröter et al., 2004; Riemann and Cliver, 2006). As they share the same terrestrial forest environment, it is not unexpected that serotypes are transmitted between rodent and reptile species. The Belem serotype that was isolated from a bat has been correlated with human infection in a previous survey that was performed in the Amazon region (Loureiro et al., 2010). The involvement of free-ranging wildlife in the transmission and dissemination of Salmonella spp. to humans and other animals remains poorly understood, but a variety of Salmonella serotypes have been isolated from both healthy and sick bats (Mühldorfer, 2013). The geographic and ecological conditions that arise from the low levels of basic sanitation in the area promote the persistence of Salmonella spp. in the Amazon ecosystem (Loureiro et al., 2010), and wildlife may contribute to its dissemination.

\section{Conclusions}

In conclusion, taking into account the different $\mathrm{VG}$ profiles and antimicrobial resistance patterns of $E$. coli strains isolated from individuals living in natural areas with dissimilar degrees of anthropization, the results obtained in this study indicate that human activity might increase the presence of resistant and/or potentially pathogenic $E$. coli strains in the environment. We also found that antimicrobial resistance is not limited to areas with greater anthropogenic impact. Regarding Salmonella spp., we observed that in natural environments, free-ranging wildlife might contribute to a cycle of perpetuation and dissemination of these bacterial species. Although the impact of these potential pathogens on wildlife is unknown, bacteria isolated from free-ranging wild animals may provide relevant information about environmental health and should therefore be more deeply studied.

\section{Acknowledgments}

The authors would like to thank all of those who contributed to the sampling of the animals and to $\mathrm{PhD}$ Fabiana Toshie de Camargo Konno, Cleide Marques da Silva Santana and Suzana Maria Bezerra for technical assistance. Renata de Oliveira Lovine was awarded financial support from CAPES.

\section{References}

Bardiau M, Grégoire F, Muylaert A et al. (2010) Enteropathogenic (EPEC), enterohaemorragic (EHEC) and verotoxigenic (VETC) Escherichia coli in wild cervids. J Appl Microbiol 109:2214-2222.

Bélanger L, Garenaux A, Harel J et al. (2011) Escherichia coli from animal reservoirs as a potential source of human extraintestinal pathogenic $E$. coli. FEMS Immunol Med Microbiol 62:1-10.

Clermont O, Bonacorsi S, Bingen E (2000) Rapid and simple determination of the Escherichia coli phylogenetic group. Appl Environ Microbiol 66:4555-4558. 
Clinical and Laboratory Standards Institute (2013a) Performance standards for antimicrobial susceptibility testing. Twentythird ed. document M100-S23. CLSI, Wayne.

Clinical and Laboratory Standards Institute (2013b) Performance standards for antimicrobial susceptibility testing; approved standard: Eighteenth international supplement document VET01-S2. CLSI, Wayne.

Costa PM, Loureiro L, Matos AJF (2013) Transfer of multidrug-resistant bacteria between intermingled ecological niches: the interface between humans, animals and the environment. Int J Environ Res Public Health 10:278-294.

Diard M, Garry L, Selva M et al. (2010) Pathogenicity-associated islands in extraintestinal pathogenic Escherichia coli are fitness elements involved in intestinal colonization. J Bacteriol 192:4885-4893.

Escobar-Páramo P, Le Menac'h A, Le Gall T et al. (2006) Identification of forces shaping the commensal Escherichia coli genetic structure by comparing animal and human isolates. Environ Microbiol 8:1975-1984.

Gannon VP, Rashed M, King RK et al. (1993) Detection and characterization of the eae gene of Shiga-like toxin-producing Escherichia coli using polymerase chain reaction. J Clin Microbiol 5:1268-1274.

Gordon DM, Cowling A (2003) The distribution and genetic structure of Escherichia coli in Australian vertebrates: host and geographic effects. Microbiology 149:3575-3586.

Gunzburg ST, Tornieporth NG, Riley LW (1995) Identification of enteropathogenic Escherichia coli by PCR-bases detection of the bundle-forming pilus gene. J Clin Mirobiol 33:1375-1377.

Hoelzer K, Switt AIM, Wiedmann M (2011) Animal contact as a source of human non-typhoidal salmonellosis. Vet Res 42:1-27.

Johnson JR, Kaster N, Kuskowski MA et al. (2003) Identification of urovirulence traits in Escherichia coli by comparison of urinary and rectal E. coli isolates from dogs with urinary tract infection. J Clin Microbiol 41:337-345.

Johnson JR, Stell AL (2000) Extended virulence genotypes of Escherichia coli strains from patients with urosepsis in relation to phylogeny and host compromise. J Infect Dis 181:261-272.

Johnson JR, Stell AL, Delavari P et al. (2001) Phylogenetic and pathotypic similarities between Escherichia coli isolates from urinary tract infections in dogs and extraintestinal infections in humans. J Infect Dis 183:897-906.

Johnson TJ, Jordan D, Kariyawasam S et al. (2010) Sequence analysis and characterization of a transferable hybrid plasmid encoding multidrug resistance and enabling zoonotic potential for extraintestinal Escherichia coli. Infect Immun 78:1931-1942.

Le Bouguenec C, Archambaud M, Labigne A (1992) Rapid and specific detection of the pap, afa, and $s f a$ adhesin-encoding operons in uropathogenic Escherichia coli strains by polymerase chain reaction. J Clin Microbiol 30:1189-1193.
Loureiro ECB, Marques NDB, Ramos FLP et al. (2010) Salmonella serovars of human origin identified in Pará State, Brazil from 1991 to 2008. Rev Pan-Amaz Saude 1:93-100.

Maynard C, Bekal S, Sanschagrin F et al. (2004) Heterogeneity among virulence and antimicrobial resistance gene profiles of extraintestinal Escherichia coli isolates of animal and human origin. J Clin Microbiol 42:5444-5452.

Michael GB, Simoneti R, Costa M et al. (2003) Comparison of different selective enrichment steps to isolate Salmonella $\mathrm{sp}$. from feces of finishing swine. Braz J Microbiol 34:138-142.

Mühldorfer K (2013) Bats and bacterial pathogens: a review. Zoonoses Public Health 60:93-103.

Obwegeser T, Stephan R, Hofer E et al. (2012) Shedding of foodborne pathogens and microbial carcass contamination of hunted wild ruminants. Vet Microbiol 159:149-154.

Olsvik O, Rimstad E, Hornes E et al. (1991) A nested PCR followed by magnetic separation of amplified fragments for detection of Escherichia coli Shiga-like toxin genes. Mol Cell Probes 5:429-435.

Östblom A, Adlerberth I, Wold AE et al. (2011) Pathogenicity island markers, virulence determinants malX and usp, and the capacity of Escherichia coli to persist in infants' commensal microbiotas. Appl Environ Microbiol 77:2303-2308.

Pollard DR, Johnson WM, Lior H et al. (1990) Rapid and specific detection of verotoxin genes in Escherichia coli by the polymerase chain reaction. J Clin Microbiol 28:540-545.

Rabinowitz P, Scotch M, Conti L (2009) Human and animal sentinels for shared health risks. Vet Ital 45:23-24.

Riemann HP, Cliver DO (2006) Foodborne infections and intoxications. Academic Press, San Diego.

Rwego IB, Isabirye-Basuta G, Gillespie TR et al. (2008) Gastrointestinal bacterial transmission among humans, mountain gorillas, and livestock in Bwindi Impenetrable National Park, Uganda. Conserv Biol 22:1600-1607.

Schröter M, Roggentin P, Hofmann J et al. (2004) Pet snakes as a reservoir for Salmonella enterica subsp. diarizonae (Serogroup IIIb): a prospective study. Appl Environ Microbiol 70:613-615.

Schwarz S, Silley P, Simjee S et al. (2010) Editorial: assessing the antimicrobial susceptibility of bacteria obtained from animals. J Antimicrob Chemother 65:601-604.

Smith JL, Fratamico PM, Gunther NW (2007) Extraintestinal pathogenic Escherichia coli. Foodborne Pathog Dis 4:134-163.

Tenaillon O, Skurnik D, Picard B et al. (2010) The population genetics of commensal Escherichia coli. Nat Rev Microbiol 8:207-217.

Trabulsi LR, Keller R, Gomes TAT (2002) Typical and atypical enteropathogenic Escherichia coli. Emerg Infect Dis 8:508-513.

Winfield MD, Groisman EA (2003) Role of nonhost environments in the lifestyles of Salmonella and Escherichia coli. Appl Environ Microbiol 69:3687-3694.

Associate Editor: Roxane Maria Fontes Piazza

All the content of the journal, except where otherwise noted, is licensed under a Creative Commons License CC BY-NC. 